\title{
Diversity and dynamics of the minichromosomal karyotype in Trypanosoma brucei ${ }^{\text {is }}$
}

\author{
Sam Alsford ${ }^{1}$, Bill Wickstead ${ }^{1}$, Klaus Ersfeld *, Keith Gull \\ School of Biological Sciences, University of Manchester, 2.205 Stopford Building, Oxford Road, Manchester M13 9PT, UK \\ Received 18 August 2000; received in revised form 28 November 2000; accepted 8 December 2000
}

\begin{abstract}
The genome of African trypanosomes contains a large number of minichromosomes. Their only proposed role is in the expansion of the parasites' repertoire of telomeric variant surface glycoprotein (VSG) genes as minichromosomes carry silent VSG gene copies in telomeric locations. Despite their importance as VSG gene donors, little is known about the actual composition of the minichromosomal karyotype and the stability of its inheritance. In this study we show, by using high-resolution pulsed-field electrophoresis, that a non-clonal trypanosome population contains an extremely diverse pattern of minichromosomes, which can be resolved into less complex clone-specific karyotypes by non-selective cloning. We show that the minichromosome patterns of such clones are stable over at least 360 generations. Furthermore, using DNA markers for specific minichromosomes, we demonstrate the mitotic stability of these minichromosomes within the population over a period of more than 5 years. Length variation is observed for an individual minichromosome and is most likely caused by a continuous telomeric growth of approximately 6 bp per telomere per cell division. This steady telomeric growth, counteracted by stochastic large losses of telomeric sequences is the most likely cause of minichromosome karyotype heterogeneity within a population. (C) 2001 Elsevier Science B.V. All rights reserved.
\end{abstract}

Keywords: Chromosome; Karyotype; Minichromosome; VSG; Telomeres; Trypanosoma brucei; Pulsed-field gel electrophoresis

\section{Introduction}

The nuclear genome of Trypanosoma brucei consists of three chromosome classes (reviewed in $[1,2]$ ). The megabase chromosomes of this diploid genome contain the expressed genes and form 11 homologous chromosome pairs [3]. The remainder of the nuclear genome consists of the intermediate chromosomes and the minichromosomes. The ploidy and function of the intermediate chromosomes is unclear, though they contain variant surface glycoprotein (VSG) gene expression sites [4]. The 100 or so minichromosomes, which are

Abbreviations: PFGE, pulsed-field gel electrophoresis; VSG, variant surface glycoprotein.

Note: Nucleotide sequence data reported in this paper are available in the GenBank ${ }^{\mathrm{TM}}$, EMBL and DDBJ-databases under the accession numbers AF294806 and AF294807.

* Corresponding author. Tel.: + 44-161-2755112; fax: + 44-1612755763.

E-mail address: k.ersfeld@man.ac.uk (K. Ersfeld).

${ }^{1}$ These two authors contributed equally to this paper. between 30 and $150 \mathrm{kbp}$ in size, constitute the group of smallest chromosomes. The sequence of a minichromosome mainly consists of a 177-bp tandem repeat, with the remaining sequence consisting of other repeats and a silent telomeric VSG gene $[5,6]$. Chromosome ends consist of typical eukaryotic telomeres. The presence of VSG genes on minichromosomes has led to the suggestion that they act as a reservoir of telomeric VSG genes, which may be transferred to expression sites by duplicative transposition $[7,8]$.

The organisation of megabase chromosomes and minichromosomes in the interphase nucleus of the cultured procyclic trypanosome and their subsequent behaviour during mitosis has been extensively characterised by fluorescence in situ hybridisation $[9,10]$. During mitosis minichromosomes are associated with the mitotic spindle and disruption of the spindle using the microtubule-depolymerising drug rhizoxin results in non-segregation of minichromosomes. The analysis of minichromosome segregation at a population level by FISH revealed the separation of the total 
minichromosomal population into two equally sized daughter populations, suggesting a high degree of fidelity of inheritance. There remains the problem of envisaging how so many minichromosomes can be segregated on a small spindle that does not exhibit large numbers of microtubules or kinetochores. A model has been proposed to explain how a faithful segregation of large numbers of minichromosomes could occur along the central mitotic spindle [11]. In this model, minichromosomes are assumed to form lateral associations with the spindle microtubules instead of the terminal associations seen in classical kinetochore-mediated chromosome segregation.

Although the available evidence on a population level suggests that the minichromosomes are segregated with a certain degree of fidelity, these observations are not sufficient to make exact statements on segregation accuracy $[10,12]$. In order to achieve this it is essential to analyse the fate of individual minichromosomes over time in a defined population of cells.

In this study, we used high-resolution pulsed-field gel electrophoresis (PFGE) to analyse the karyotype pattern of minichromosomes in clonal cell lines. In addition to employing probes detecting the entire minichromosomal complement of a cell, we also developed markers for specific minichromosomes in this population. We demonstrate that minichromosomes are accurately segregated over time. We also describe a high degree of minichromosomal diversity in non-clonal populations and provide evidence that this heterogeneity is unlikely to be due to non-disjunction or chromosome loss but rather is a consequence of size variations of individual chromosomes within the cells of the population.

\section{Materials and methods}

\subsection{Limiting dilution cloning of procyclic T. brucei}

Our laboratory stock of $T$. brucei strain 427 has been maintained in culture over a period of 10 years, during which time it has not been cloned. Therefore, it was thought that the cloning of this population might reveal the degree of fidelity with which the minichromosomes are segregated by analysing the stability of the karyotype pattern. Sets of primary clones were produced by non-selective limiting dilution cloning from a contemporary laboratory stock of EATRO 427 (1998 and 2000) and from a sample that had been frozen in liquid nitrogen in our laboratory in 1993. All primary clones were subsequently recloned to produce secondary clone pairs, with each pair corresponding to a specific primary clone.

Procyclic stage cells of the culture-adapted 427 strain were cloned in the absence of selective pressure by limiting dilution cloning in conditioned media. Conditioned media was prepared as follows. Procyclics were grown at $28^{\circ} \mathrm{C}$ in SDM79 [13] supplemented with $10 \%$ $(\mathrm{v} / \mathrm{v})$ foetal calf serum (FCS) to a density of approximately $1 \times 10^{7}$ cells $\mathrm{ml}^{-1}$. The culture was pelleted and then was supplemented with $10 \%(\mathrm{v} / \mathrm{v}) \mathrm{FCS}$, filter sterilised through a $0.22 \mu \mathrm{m}$ filter, stored at $4^{\circ} \mathrm{C}$ and used within a week of preparation.

For cloning, the culture was diluted to a final density of about one cell ml-1 in $20 \mathrm{ml}$ conditioned media and transferred to a 96 -well plate at $200 \mu 1$ per well. After 7 days at $28^{\circ} \mathrm{C}$, populated wells were inoculated into 10 ml SDM79 supplemented with 10\% (v/v) FCS and placed at $28^{\circ} \mathrm{C}$. Primary clones were identified by the co-ordinate of their source well (letter, row; number, column) and secondary clones were identified by the source primary clone and their source well. Therefore, primary clone $\mathrm{C} 10$ was isolated from well $\mathrm{C} 10$ of a 96-well plate, while secondary clone C10D4 was isolated from well D4 following the re-cloning of primary clone $\mathrm{C} 10$.

\subsection{Pulsed-field gel electrophoresis}

Chromosome blocks were prepared as earlier described [3]. PFGE was carried out in a contour-clamped homogeneous electric field apparatus (CHEF DRII or CHEF Mapper, BioRad). Chromosome separation was performed in 1 or $1.5 \%$ agarose (PFGE grade, Amersham-Pharmacia Biotech) in $90 \mathrm{mM}$ Tris-borate $\mathrm{pH}$ 8.2, $0.2 \mathrm{mM}$ EDTA $(1 \times \mathrm{TB}[0.1] \mathrm{E})$ at constant temperature with buffer circulation. Specific conditions are described in the figure legends.

Concurrent electrophoresis of $\lambda$-concatamers (Promega) and Eco RI/HindIII digested $\lambda$-phage (Appligene) allowed T.brucei chromosome sizes to be estimated. Following separation, chromosomes were stained in $2.5 \mu \mathrm{g} \mathrm{ml}^{-1}$ ethidium bromide for $30 \mathrm{~min}$ and destained in distilled water for $30 \mathrm{~min}$. Images were digitally captured under $300 \mathrm{~nm}$ UV illumination.

\subsection{Southern blot analysis}

Separated chromosomes were depurinated in $0.25 \mathrm{M}$ $\mathrm{HCl}$ for $20 \mathrm{~min}$, denatured and transferred onto nylon membranes by standard procedures. DNA probes for the 177-bp minichromosome repeat and the telomere repeat oligonucleotide was labelled with digoxigenin as described $[10,14]$. The 177 bp repeat-specific probe was hybridised in EasyHyb-buffer (Roche Diagnostics) at $40^{\circ} \mathrm{C}$ overnight and the final washes were carried out in $15 \mathrm{mM} \mathrm{NaCl}, 1.5 \mathrm{mM}$ sodium citrate $(0.1 \times \mathrm{SSC}) / 0.1 \%$ $(\mathrm{w} / \mathrm{v}) \mathrm{SDS}$ at $60^{\circ} \mathrm{C}$. The telomere-specific probe was hybridised at $37^{\circ} \mathrm{C}$ for $3 \mathrm{~h}$ and the final washes were carried out at $50^{\circ} \mathrm{C}$ in $0.5 \times \mathrm{SSC} / 0.1 \%(\mathrm{w} / \mathrm{v}) \mathrm{SDS}$. To optimise detection of the less abundant probes, we used 
UV-nicking ( $80 \mathrm{~mJ}$ at $250 \mathrm{~nm}$ ) instead of depurination and transfer under alkaline conditions in $0.4 \mathrm{M} \mathrm{NaOH}$, $1.5 \mathrm{M} \mathrm{NaCl}$. Blots were developed with an anti-digoxigenin antibody (Fab-fragment) conjugated to alkaline phosphatase and CSPD as a chemiluminescent substrate (all from Roche Diagnostics).

\subsection{Isolation of minichromosomal DNA}

Chromosome plugs were prepared as described above. Electrophoresis was conducted in a CHEF DRII unit (BioRad) at $12^{\circ} \mathrm{C}$ and $200 \mathrm{~V}$ for $35 \mathrm{~h}$ with a constant electrode switching time of $300 \mathrm{~s}$. Gels were $1 \%$ LMP agarose (SeaPlaqueGTG; FMC BioProducts) in $1 \times \mathrm{TB}(0.1)$ E. DNA was stained with $1 \mu \mathrm{g} \mathrm{ml} \mathrm{m}^{-1}$ ethidium bromide, destained and minichromosomes were excised with a scalpel under $300 \mathrm{~nm} \mathrm{UV}$ illumination.

Gel fragments were equilibrated in $10 \mathrm{mM}$ Bis-Tris$\mathrm{HCl}, 1 \mathrm{mM}$ EDTA, pH 6.5 and minichromosomal DNA was released by digestion with $\beta$-agarase I (New England BioLabs) for $16 \mathrm{~h}$ at $40^{\circ} \mathrm{C}$. The salt content of the solution was then adjusted with $10 \times$ restriction buffer H (Roche Diagnostics) and the DNA digested with restriction endonucleases Eco RI and XhoI for $5 \mathrm{~h}$ at $37^{\circ} \mathrm{C}$. The rational of choosing these enzymes was the fact that they do not cut within the $177 \mathrm{bp}$ repeat. Digested DNA was recovered by ethanol precipitation and fragments of size $2-10 \mathrm{kbp}$ were selected by gel electrophoresis and gel-purified (QIAquick gel extraction kit; Qiagen). DNA fragments were then ligated into the cloning vector pBluescript SK II $(+)$ (Stratagene). Positive transformants were selected at random and the plasmid DNAs screened for hybridisation to minichromosomal DNA immobilised on membranes. Clones of interest were sequenced using the BigDye kit (Applied Biosystems).

The minichromosomal RNA pol I promoter as described by Zomerdijk and Borst [12] was obtained by PCR using the oligonucleotides (5'-ACCGTAGCTCTCCACCCA) and (5'-GCGCCTACGAGCTGTGAA).

\section{Results}

\subsection{Clone-specific minichromosome PFGE karyotypes}

PFGE of an EATRO 427 laboratory stock of procyclic trypanosomes revealed a characteristic pattern of megabase chromosomes and intermediate chromosomes (Fig. 1). Under these PFGE conditions, the minichromosome population was present as an intensely fluorescent band at the bottom of the gel, when stained with ethidium bromide.
We subjected this trypanosome population, which has been grown virtually constantly and has not been cloned within a 10-year window, to non-selective cloning by limiting dilution. Analysis of the resulting clones presented essentially the same pattern for all chromosomes at this level of resolution. Thus, under these gel conditions the karyotype of the source population and all the resulting clones was indistinguishable. This is consistent with the earlier findings, also based on PFGE analysis, that demonstrated the megabase chromosome karyotype of T.brucei to be stable over time in mitotically replicating cells [15].

In order to analyse the minichromosome population in more detail, we developed PFGE conditions that allowed separation of the minichromosomes at high resolution. We estimated this level of PFGE resolution to be $\pm 1 \mathrm{kbp}$. We analysed the above clones using these conditions and we found a clear clone-specific PFGE karyotype for minichromosomes (Fig. 2B). In contrast to the specific patterns of the clones, it was almost impossible to distinguish between individual bands of minichromosomes in the uncloned source population. The complexity in the source population pattern was, therefore, resolved into individual simpler clone-specific patterns. This complexity of the original source population and the specific patterns resulting from cloning was also seen when probing corresponding Southern blots with a telomeric oligonucleotide or with a 177 bp repeat DNA probe (Fig. 2B).

We repeated this analysis with an aliquot of the same 427 strain, which has been kept frozen in the laboratory

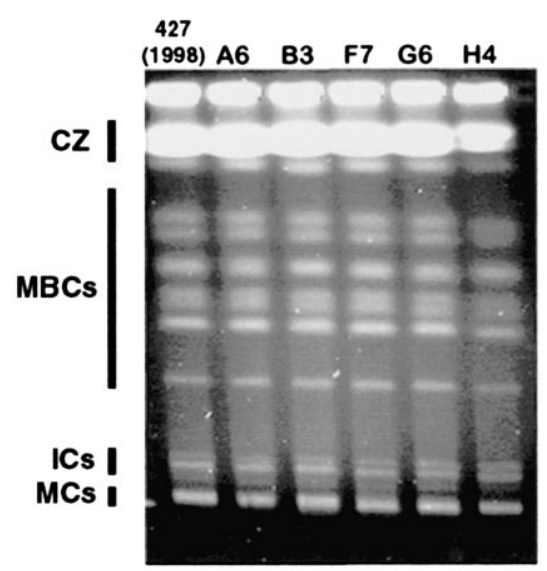

Fig. 1. The molecular karyotypes of the smallest megabase chromosomes of the 427 (1998) population and primary clones thereof. The patterns of source population and clones are identical. The compression zone (CZ) contains unresolved megabase chromosomes. The different classes of chromosomes separated are indicated (MBCs, small megabase chromosomes (1-2 Mbp); ICs, intermediate chromosomes (200-400 kbp); MCs, minichromosomes (30-150 kbp)) Separation was carried out on a CHEF DRII (BioRad) in $1 \%$ PFGE agarose at $2.4 \mathrm{~V} \mathrm{~cm}^{-1}$ in $1 \times \mathrm{TB}(0.1) \mathrm{E}$ at a constant $12^{\circ} \mathrm{C}, 1400-700$ $\mathrm{s}$ switching time ramped linearly over $144 \mathrm{~h}$. The gel was stained in ethidium bromide. 


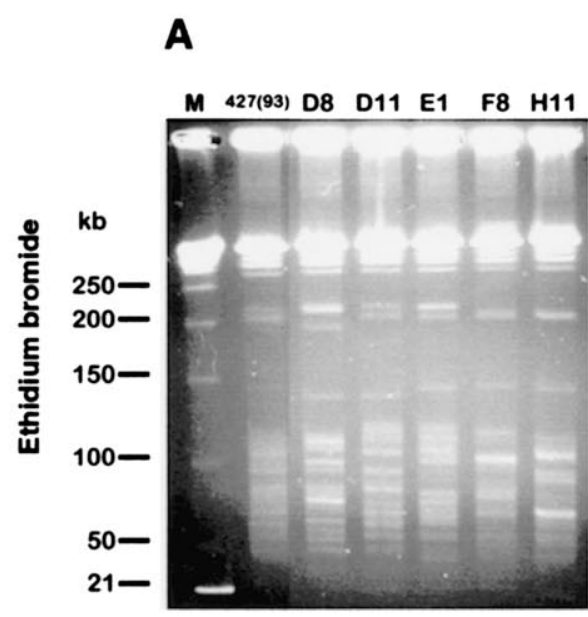

B
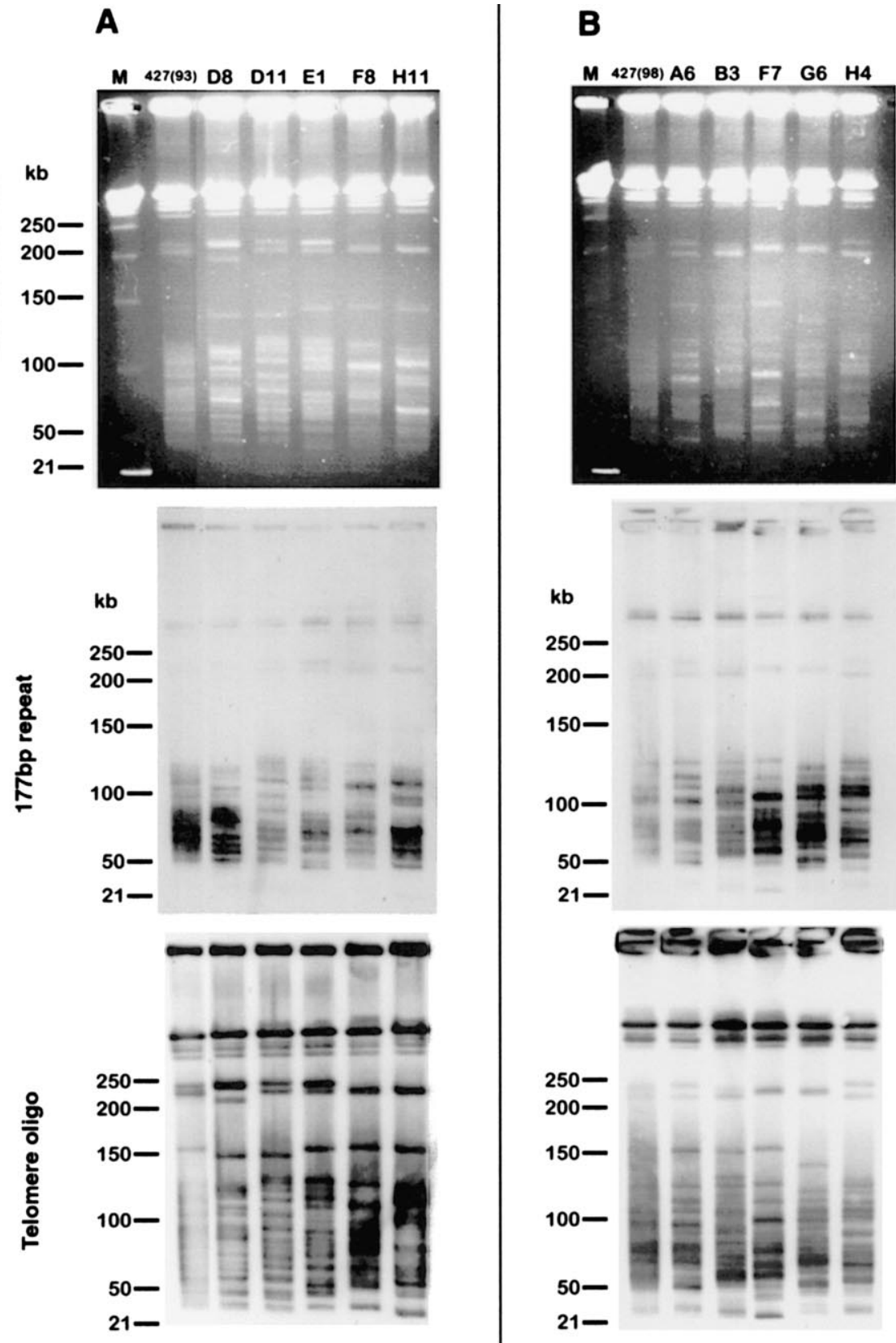

Fig. 2. The minichromosome molecular karyotypes of primary clones derived from the 1993 (A) and 1998 (B) source populations reveal that the original populations contain complex patterns of minichromosome molecular karyotypes reflected in the different patterns of each derived clone. Separated chromosomes were stained with ethidium bromide and subsequently blotted and hybridised with the 177 bp minichromosome-specific repeat and the telomere oligonucleotide. Lane M, $50 \mathrm{~kb} \lambda$-ladder and Eco RI/HindIII digested $\lambda$. Separation was carried out on a CHEF DRII (BioRad) in $1.5 \%$ PFGE agarose at $5.3 \mathrm{~V} \mathrm{~cm}^{-1}$ in $1 \times \mathrm{TB}(0.1) \mathrm{E}$ at a constant $12^{\circ} \mathrm{C}$, switching time 10 and $20 \mathrm{~s}$ for $25 \mathrm{~h}$ each.

since 1993 (Fig. 2A). The result was essentially the same, each clone showed a specific individual minichromosome pattern, which was less complex than that of the source population. None of the clones $(n=18)$ from any of the populations studied could be matched to any other clone.

It is also of interest that the heterogeneity of chromosomes within the population included the intermediatesized chromosomes (between 200 and $400 \mathrm{kbp}$ in size). Both the 1993 and 1998 derived clones revealed at least three different karyotypes with respect to intermediate chromosomes. Clones D8, D11/E1 and F8/H11 (derived from the 1993 population) and clones A6/H4, B3 and F7/G6 (derived from the 1998 population) each formed three distinct groups based on intermediate chromosome banding pattern.

The surprising diversity of minichromosome patterns within a population led us to question the origins of this heterogeneity. Possible causes would include implicit diversity within populations or dynamic events 
such as random loss or gain of chromosomes during mitosis (non-disjunction events) or size variation of individual chromosomes.

\subsection{Origin and stability of the individual minichromosomal karyotypes}

The stability of the minichromosome karyotypes was assessed by producing secondary clone pairs from the
1998 population and maintaining them in culture for 120 days (equivalent to approximately 360 generations).

A comparison of the minichromosome PFGE karyotypes of three secondary clone pairs (derived from primary clones A6, F7, F8) at day 0 and 120 is shown in Fig. 3. This comparison revealed that the minichromosome karyotypes were stable on secondary cloning and further culturing for 120 days. Each identifiable band was maintained through time, suggesting that the

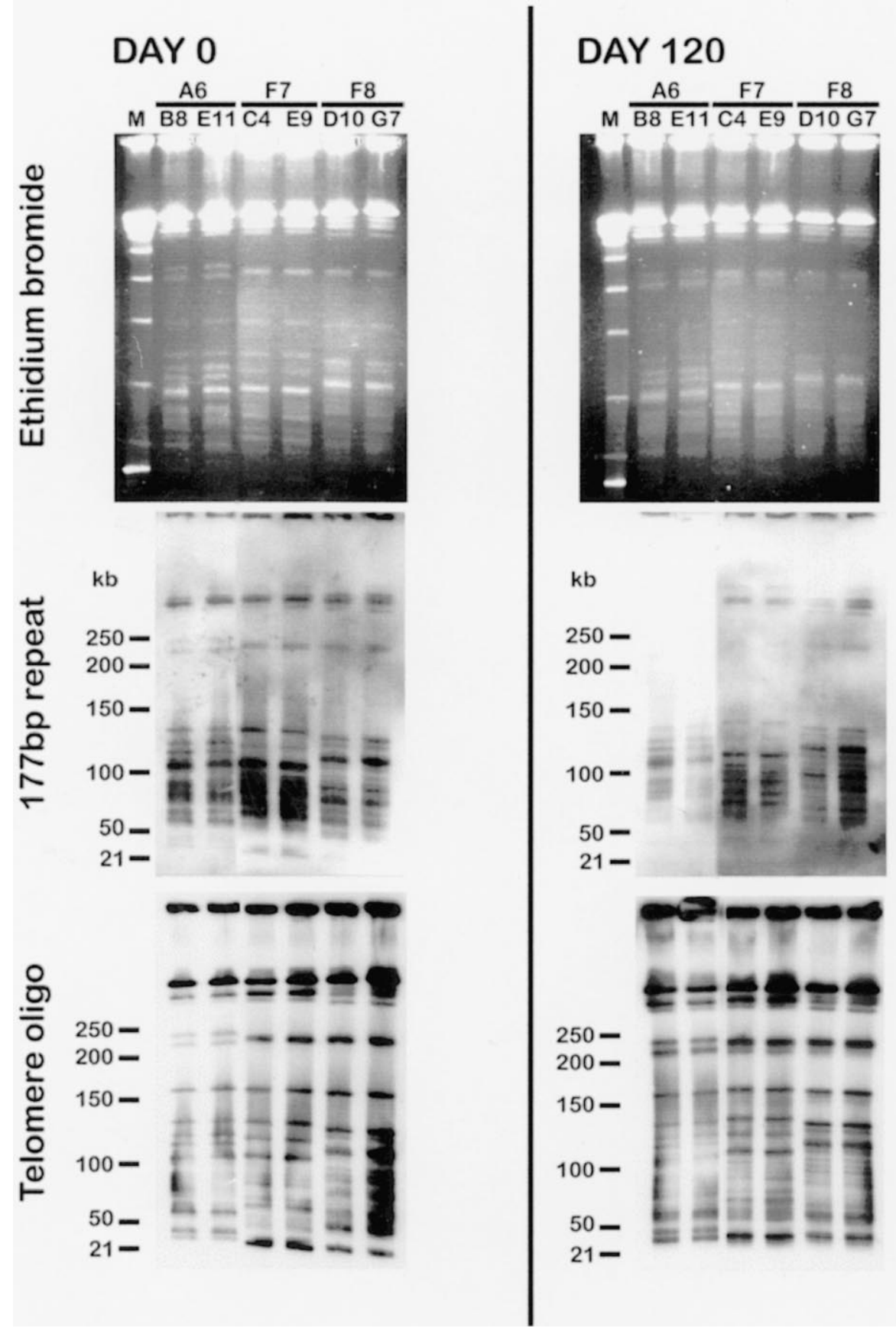

Fig. 3. Karyotypes of pairs of secondary clones derived from primary clones described in Fig. 2 were analysed by ethidium bromide staining and Southern blotting. The minichromosome molecular karyotypes are stable over 120 days, which is equivalent to 360 generations. Hybridisation of the transferred chromosomes with the $177 \mathrm{bp}$ minichromosome repeat and the telomere oligonucleotide revealed no changes in the MC molecular karyotype during 120 days of growth. Lane M, $50 \mathrm{~kb} \lambda$-ladder and Eco RI/HindIII digested $\lambda$. See legend on Fig. 2 for separation conditions. 


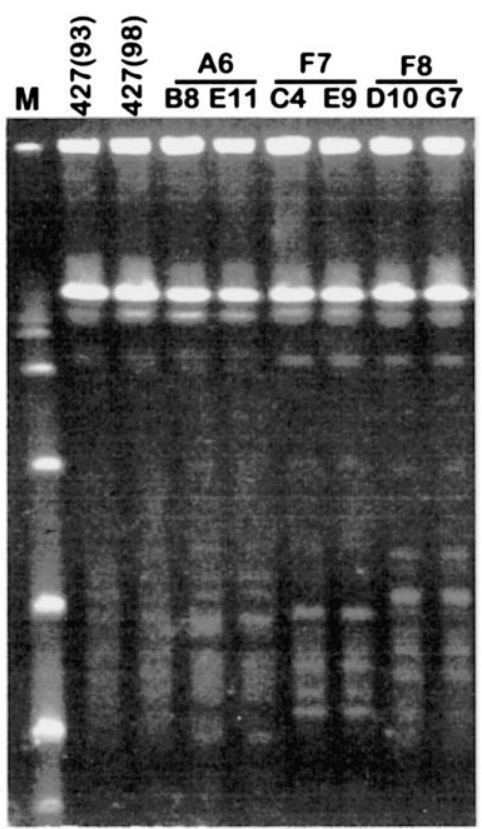

Ethidium bromide
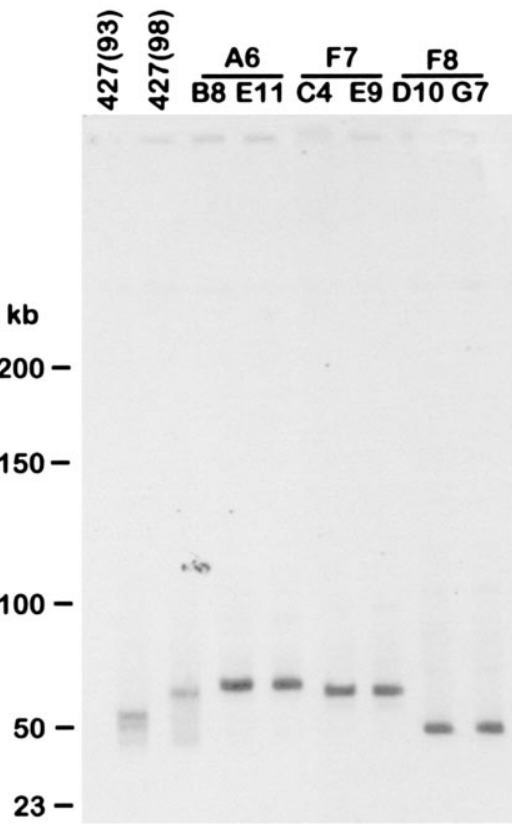

VSG S8

Fig. 4. Analysis of an individual minichromosome using the VSG S8 probe. The two lanes on the left side of each panel show the ethidium bromide and VSG S8 hybridisation pattern of the non-clonal population 427 (frozen in 1993) and 427 (1998). The remaining lanes show the ethidium bromide pattern and hybridisation pattern of pairs of clones with the VSG S8 probe. Note the trailing of the signal in the non-clonal population and the resolution into single bands in the clonal populations. Electrophoresis was conducted in a CHEF Mapper unit (BioRad) at $12^{\circ} \mathrm{C}$ and $4.6 \mathrm{~V} \mathrm{~cm}^{-1}$ for $42 \mathrm{~h}$. The included angle between electrode states was $120^{\circ}$ and the electrode switching time was ramped linearly from 8 to 15 s. Gels were $1 \%$ SeaKem Gold agarose (FMC BioProducts) in $1 \times \mathrm{TB}(0.1) \mathrm{E}$. M, marker.

minichromosomes of a population were mitotically stable at this level of resolution. The stability was reflected by all three analytical approaches, the ethidium bromide patterns were identical as were the Southern blots probed with the 177-bp repeat DNA probe and with a telomeric oligonucleotide.

\subsection{Stability and dynamics of individual minichromosomes}

Even with PFGE conditions optimised to separate minichromosomes, it is very difficult to analyse the dynamics of an individual chromosome within the total population of approximately 100 minichromosomes per trypanosome. Therefore, we identified DNA sequences that occur only on a very small number or even an individual minichromosome. From a genomic library constructed from gel-extracted and restricted minichromosomal DNA, we isolated DNA fragments and selected clones suitable as minichromosomal markers based on hybridisation to PFGE-blots. Several DNA clones were identified and some used for the experiments described below. Sequence analysis of these DNA clones showed two (S8, G4) to be minichromosomal VSG genes and one (ingi3) to be an ingi retroposon element.

The VSG S8 was used to probe PFGE blots of non-clonal $T$. brucei 427 source populations derived from the 1993 frozen stock and from the 1998 lab maintained stock (Fig. 4). Although some prominent bands were detected in each source population (50 and $55 \mathrm{kbp}$ in 427(93) and $70 \mathrm{kbp}$ in 427(98)), there was a wide spread of the hybridisation signal and a clearly visible trail ranging from 30 to $70 \mathrm{kbp}$. In addition, there was a distinct difference in pattern between the 1993 and 1998 source populations. We then analysed the clones of the 1998 population (Fig. 4, clones as described in Fig. 3). In the clonal populations, only single discrete bands were observed. The size range of these bands fell within the range of the hybridisation signals observed in the parental 1998 source population. This indicated that individual minichromosomes in non-clonal populations display a high degree of size polymorphism, which can be resolved by cloning. Neither the 1993/1998 populations nor any of the derived clones $(n=29$, exemplified by the three pairs of clones shown in Fig. 4) had lost the VSG, indicating a high degree of mitotic stability over long periods of time.

To investigate the dynamics of size-polymorphism of individual minichromosomes, we compared clones before and after 120 days of growth in culture (Fig. 5). Here, it is important to note that the cells, which were grown for 120 days were not recloned prior to analysis (preparation of PFGE plugs) but reflect the full heterogeneity which was acquired over that period of time. After 120 days (equivalent to 360 generations), we 
noticed a size increase of the major band of approximately $4 \mathrm{kbp}$ in all clones analysed. This corresponds to an increase of about $11 \mathrm{bp}$ per generation. In addition, the band after 120 days was less defined and a signal trailed from this major band over a range of approximately $20 \mathrm{kbp}$ in the case of the clones A6B8 and F7C4 and $2-3 \mathrm{kbp}$ for clone F8D10.

It is interesting to note that we always observed only a single band in the minichromosomal population carrying the VSG S8 gene. This may be an evidence for a single copy minichromosome. If, however, there are multiple homologous chromosomes within this band then chromosome dynamic events on homologous chromosomes must be co-ordinated or operate in parallel. In order to address the question of the frequency at which minichromosomal sequence elements occur on these chromosomes, we probed CHEF-blots with a further VSG gene probe (VSG G4) and the ingi probe (ingi 3). Both sequences were recovered from the minichromosomal library as described above. On the blots, the parental strain was compared with primary clones derived from this uncloned source population (Fig. 6). In contrast to the VSG S8 gene, which appeared to be present on a single minichromosome, the
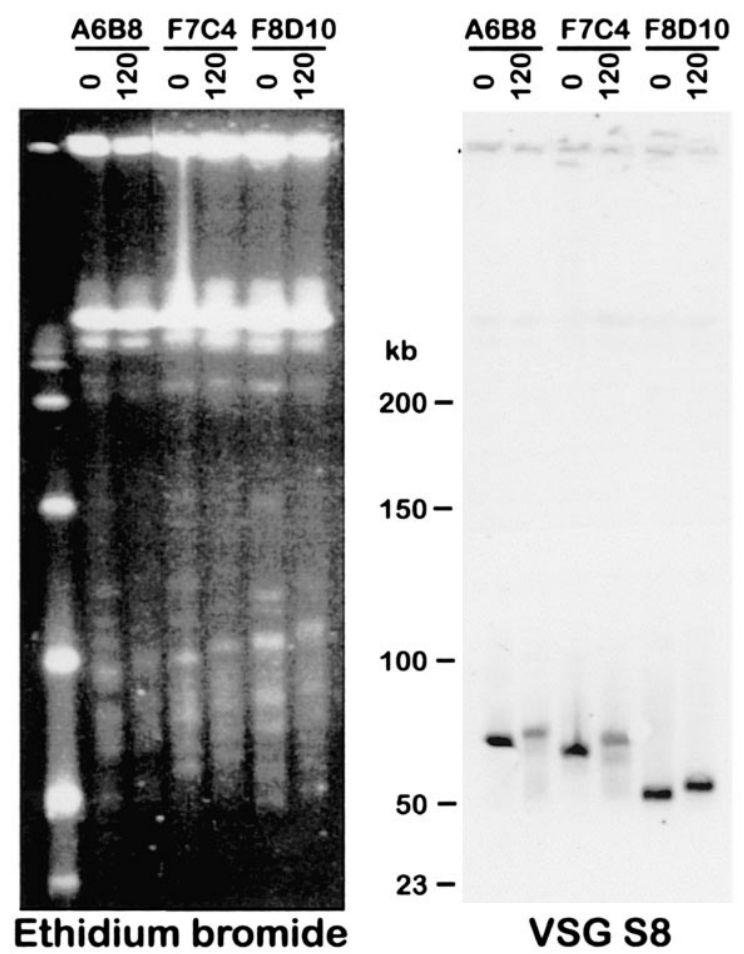

Fig. 5. Analysis of the stability of an individual minichromosome in clonal populations over time. A selection of three clones as described in Fig. 5 has been grown over 120 days and analysed by probing with the specific probe VSG S8. The left panel shows the ethidium bromide staining and the right panel the corresponding hybridisation. Note the shift of all hybridisation signals by approximately $4 \mathrm{~kb}$, but also the smear appearing underneath the major band of each clone after 120 days. See legend on Fig. 5 for separation conditions.
VSG G4 was present on multiple minichromosomes. Depending on the clone upto 4 minichromosomal bands (e.g. clone 3) could be resolved. All signals observed in the different clones fell within the range of the signal obtained for the source population. In addition, we used a rDNA promoter probe to investigate the occurrence of this element in our trypanosome lab strain. Zomerdijk et al. [12] reported an orphan rDNA promoter residing on two bands within a minichromosomal population. We confirmed the presence of this ribosomal promoter on minichromosomes in our lab strain of 427. In contrast to the observations made by Zomerdijk et al. we found, however, that the promoter occurred on at least four minichromosomes. This is likely to be due to the greater resolution of our PFGE conditions coupled with chromosomal size variability. Interestingly, the VSG and rDNA promoter containing minichromosomes cluster within a certain limited size window of the total minichromosomal population. Whereas the VSG G4 containing chromosomes are between 70 and $100 \mathrm{~kb}$ in size, the rDNA promoter containing chromosomes are only $25-60 \mathrm{~kb}$ in size. Finally, we investigated the distribution of the ingi3 element on minichromosomes. Ingis are retroposon-like elements, which have probably lost their ability to be mobile within the trypanosome genome [16,17]. They are dispersed amongst all chromosome classes in $T$. brucei. Analysis of our cloned 427-cell line revealed their presence on minichromosomes. Upto five distinct bands were resolved (e.g. clone 4). However, their size distribution is non-random. In the source population, three ingi clusters were found, ranging in size from 50 to 60,80 to 100 and 130 to $170 \mathrm{~kb}$, respectively. This clustering was not reflected in the overall ethidium bromide-staining pattern of minichromosomes (see Figs. 2 and 3). Possible explanations for the observed non-random distribution of several markers among the minichromosomal population could be an intrinsic qualitative difference between these chromosomes or the acquisition of genes or other DNA elements such as ingis by a single chromosome with subsequent duplications and size fluctuations.

\section{Discussion}

The genome of salivarian trypanosome species undergoing antigenic variation is characterised by the presence of minichromosomes [18-20]. The identification of non-transcribed telomeric VSG genes on minichromosomes led to the hypothesis that they serve as a reservoir for these genes [20]. Recent work on the prevalence of various genetic mechanisms leading to VSG expression site activation in $T$. brucei lends support to this hypothesis by showing that minichromosomes serve as major donors of telomeric VSG genes during duplicative gene conversions [7]. 

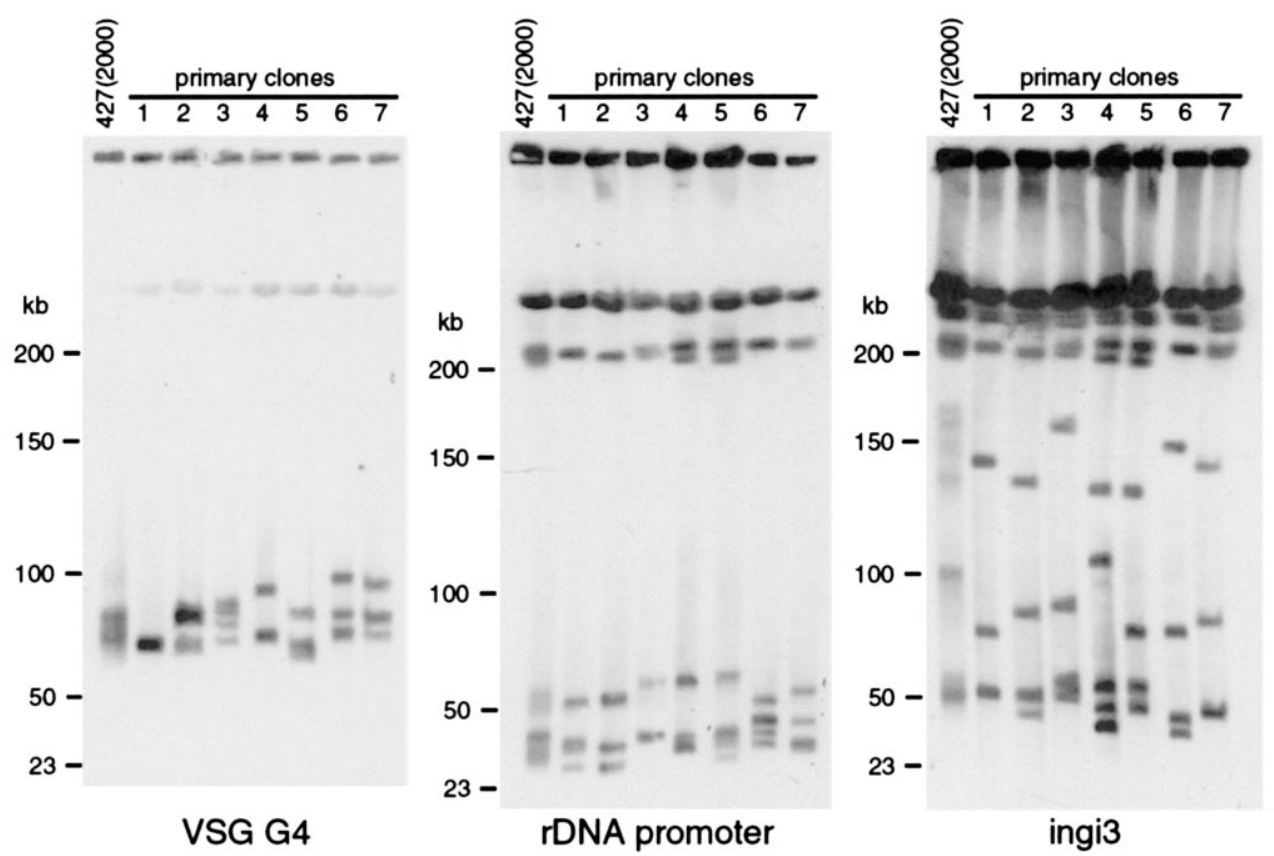

Fig. 6. Analysis of clonal populations of trypanosomes using different DNA elements found on minichromosomes (VSG-G4 gene, ribosomal promoter, ingi3). The left lane in each panel contains the DNA from the uncloned parental populations from which clones 1-7 are derived. PFGE conditions were as described in Fig. 4.

The genome of $T$. brucei contains approximately 100 minichromosomes. In order to maintain this minichromosome population and reservoir of VSG genes, a mechanism able to provide fidelity of mitotic segregation over long periods of time appears likely to exist. In an earlier study, we have shown that minichromosomes are, in principle, segregated via the typical eukaryotic chromosome segregation mechanism, which includes the dependence of segregation on an intact mitotic spindle [10]. Although possibly different in detail from the classical chromosome-spindle association, this observation provides a mechanism whereby minichromosomes can be stably segregated during mitosis [11]. Whereas these findings were based on cytological observation, this present study was designed to analyse karyotype patterns within a population over time in a more detailed manner.

In order to have defined starting populations to conduct this investigation, we cloned individual cells of the T. brucei 427 strain. Using PFGE, we showed that the pattern of megabase chromosomes was indistinguishable between clones and their source populations. However, the separation of minichromosomes at high resolution revealed clone-specific patterns. We found a high level of minichromosome karyotype heterogeneity within a population but of the 18 clones analysed none had a pattern identical to any other clone. The technique of using high-resolution PFGE of minichromosome populations to study clonality is likely to be of use in many other studies of population analysis in trypanosomes.
What could be the causes of this variation within a population? Variability as a result of sexual recombination events was unlikely because recombination as a consequence of mating [21] is thought to occur only in the natural vector, the tsetse fly and has never been described in mitotically replicating, cultivated procyclic trypanosomes [15]. Moreover, mating events would be reflected by a Mendelian inheritance pattern within the megabase pair chromosomes of clonal populations, which was not seen. Extensive loss and gain of chromosomes as a result of non-disjunction during mitosis could not have been ruled out a priori but was unlikely to occur on this scale as it would inevitably lead to extremely heterogeneous populations in terms of the total number of minichromosomes. This has never been observed. Also, our finding of the single VSG S8 chromosome band in every clone $(n=29)$ from the population argues against non-disjunction.

To analyse remaining possibilities for the basis of this karyotype heterogeneity of minichromosomes in more detail, we constructed a minichromosome-enriched genomic library and screened for DNA clones, which hybridised to distinct minichromosomes on PFGEblots. We identified one clone (S8) that hybridised to only one of the minichromosomes. Using this clone to analyse several clonal populations, we showed that (a) the marker is stably maintained in all of the clones and in their parental populations (b) the heterogeneity observed using ubiquitous DNA markers (177 bp repeat, telomere oligo) was reflected in size variations of this single chromosome and (c) over time (120 days, 360 
generations) a consistent size increase of approximately $4 \mathrm{kbp}$ was observed for the chromosome carrying this marker gene. This equals an increase in size of around $11 \mathrm{bp}$ per cell division. In addition to a size increase of the dominant band within a given population, the hybridisation pattern showed a trailing signal, indicating some cells in the population had undergone a decrease in size of this chromosome.

It has long been observed that the telomeric ends of the megabase chromosomes exhibit a linear growth rate of approximately $6-10 \mathrm{bp}$ per cell division [22,23]. A more detailed recent analysis has confirmed these findings and calculates a growth rate of about 8 bp per division [24]. These calculations of telomeric growth agree well with our observed growth rate for minichromosomes of approximately $6 \mathrm{bp}$ per telomere per cell division. A similar telomeric growth rate has been found for artificial minichromosomes [25]. The amount of size variation of the VSG-S8-carrying chromosome ( $\sim 13 \mathrm{kbp}$ per telomere) is also comparable to that observed for telomeres of megabase chromosomes $[24,26]$. Both the megabase chromosomes and the minichromosomes have identical telomere sequences $\left(\mathrm{T}_{2} \mathrm{AG}_{3}\right)_{n}$ and it is therefore very likely that the same telomerase complex, which acts on large chromosomes, also maintains minichromosomal telomeres. It has also been shown that the steady and slow growth of telomeres is occasionally counteracted by large deletions of telomeric DNA [23]. In contrast to continuous telomere growth, this 'telomere catastrophe' is a stochastic event. The most plausible explanation of the minichromosomal growth over time is, therefore, the gradual increase in telomere length. Although we cannot rule out recombination events as an additional cause of size variation in minichromosomes, our consistent observations of a steady and directional growth at a constant average rate in all of the clones examined argues against recombination as a major contributing factor.

Our investigations relate only to mitotically propagating procyclic trypanosomes. It will, therefore, be interesting to study the involvement of minichromosomes in genetic exchange occurring as a result of mating between trypanosomes in the tsetse fly. A high degree of genetic exchange of minichromosomal DNA would certainly contribute efficiently to VSG gene repertoire variability in populations. The general stability of patterns in procyclics is important as it suggests that individual minichromosomes are not retained by a potential selective pressure occurring in VSG-expressing bloodstream forms.

We have shown that an uncloned population of $T$. brucei cells exhibits a considerable heterogeneity of minichromosomal PFGE karyotypes, which is resolved on cloning into simpler clone-specific patterns. The complexity of the minichromosome karyotype of the parental populations is thus derived from a superposi- tion of a large number of simpler subpopulations. Clone-specific patterns were stable over at least 360 cell generations in culture. We have also demonstrated that a VSG gene, marking an individual minichromosome, is stably maintained in populations separated by 5 years and also inherited by all clones derived from these populations. This indicates that individual minichromosomes are replicated efficiently during the cell cycle and are segregated with high fidelity during mitosis. Analysis of this individual chromosome over time revealed that long-term size variations of chromosomes are the most likely cause of minichromosome karyotype complexity. The origin of these variations is most likely telomeric size fluctuations and shows that telomere dynamics of minichromosomes and megabase chromosomes in T. brucei are comparable.

An earlier report suggested that minichromosomes are stably inherited [12]. In that investigation, a minichromosome was tagged by recombining a neomycin resistance gene downstream of an orphan, but functional, ribosomal promoter. When cells were grown in the absence of neomycin, antibiotic resistance was nevertheless retained over at least 130 generations. However, another report, where a similar tagged minichromosome was used (hygromycin resistance was used instead of neomycin resistance) showed that the hygromycin gene was lost with a half-life of approximately 14 generations in the absence of selection [27]. Even though both studies used different assays, it is difficult to provide a resolution of the different outcomes. However, both studies were based on the behaviour of an artificially modified minichromosome, which was forced to be transcriptionally active, a situation that may not normally be found on these chromosomes. Our data suggests that endogenous, unmodified minichromosomes are mitotically stable.

Finally, we turn to the question of minichromosomal ploidy. Minichromosomes have often been regarded as being aneuploid. Also, there is one reference that describes the presence of an orphan ribosomal promoter on two minichromosomal bands [12]. These authors carefully rehearsed that one possible explanation for observation was the presence of two homologous minichromosomes. When we re-examined our 427 strain of $T$. brucei for the presence of the ribosomal promoter on minichromosomes, we found under PFGE conditions optimised for minichromosomal separation, at least four distinct bands carrying this promoter. Using two additional minichromosomal VSG probes (S8 and G4), we detected one and four bands, respectively, for these probes. In the case of the ingi3 retroposon-like element, which was also found on minichromosomes, we distinguished at least five bands within the minichromosomal size range. We, therefore, also conclude that there is currently insufficient evidence to decide the question of minichromosomal ploidy within this complex genome. 


\section{Acknowledgements}

This work was supported by BBSRC studentships to S.A. and B.W. and a Wellcome Trust Programme and Equipment Grant.

\section{References}

[1] El-Sayed N, Hedge P, Quackenbusch J, Melville SE, Donelson JE. The African trypanosome genome. Int $\mathbf{J}$ Parasitol 2000;30:329-45.

[2] Ersfeld K, Melville SE, Gull K. Nuclear and genome organisation of Trypanosoma brucei. Parasitol Today 1999;15:58-63.

[3] Melville SE, Leech V, Gerrard CS, Tait A, Blackwell JM. The molecular karyotype of the megabase chromosomes of Trypanosoma brucei and the assignment of chromosome markers. Mol Biochem Parasitol 1998;94:155-73.

[4] van der Ploeg LHT, Cornelissen AWCA, Michels PAM, Borst P. Chromosome rearrangements in Trypanosoma brucei. Cell 1984;39:213-21

[5] Weiden M, Osheim YN, Beyer AL, Van der Ploeg LHT. Chromosome structure: DNA nucleotide sequence elements of a subset of the minichromosomes of the protozoan Trypanosoma brucei. Mol Cell Biol 1991;11:3823-34.

[6] Williams RO, Young JR, Majiwa PAO. Genomic environment of $T$. brucei VSG genes: presence of a minichromosome. Nature 1982;299:417-21.

[7] Robinson NP, Burman N, Melville SE, Barry JD. Predominance of duplicative VSG gene conversion in antigenic variation in African trypanosomes. Mol Cell Biol 1999;19:5839-46.

[8] van der Ploeg LHT, Schwartz DC, Cantor CR, Borst P. Antigenic variation in Trypanosoma brucei analyzed by electrophoretic separation of chromosome-sized DNA molecules. Cell 1984;37:77-84

[9] Chung HMM, Shea C, Fields S, Taub RN, Van Der Ploeg LHT, Tse DB. Architectural organization in the interphase nucleus of the protozoan Trypanosoma brucei: location of telomeres and mini-chromosomes. EMBO J 1990;9:2611-9.

[10] Ersfeld K, Gull K. Partitioning of large and minichromosomes in Trypanosoma brucei. Science 1997;276:611-4.

[11] Gull K, Alsford S, Ersfeld K. Segregation of minichromosomes in trypanosomes: implications for mitotic mechanisms. Trends Microbiol 1998;6:319-23.
[12] Zomerdijk J, Kieft R, Borst P. A ribosomal RNA gene promoter at the telomere of a mini-chromosome in Trypanosoma brucei. Nucl Acids Res 1992;20:2725-34.

[13] Brun R, Schönenburger M. Cultivation and in vitro cloning of procyclyc forms of Trypanosoma brucei in a semi-defined medium. Acta Trop 1979;36:289-92.

[14] Ogbadoyi E, Ersfeld K, Robinson D, Sherwin T, Gull K. Architecture of the Trypanosoma brucei nucleus during interphase and mitosis. Chromosoma 2000;108:501-13.

[15] Tait A, Buchanan N, Hide G, Turner CMR. Self-fertilisation in Trypanosoma brucei. Mol Biochem Parasitol 1996;76:31-42.

[16] Braun R, Behrens K, Glauser A, Brun R. Evolution of the retrotransposons TRS/ingi and of the tubulin genes in trypanosomes. Acta Trop 1992;52:175-87.

[17] Kimmel BE, Olemoiyoi OK, Young JR. Ingi, a 5.2-kb dispersed sequence element from Trypanosoma brucei that carries half of a smaller mobile element at either end and has homology with mammalian LINEs. Mol Cell Biol 1987;7:1465-75.

[18] Dickin SK, Gibson WC. Hybridisation with a repetitive DNA probe reveals the presence of small chromosomes in Trypanosoma vivax. Mol Biochem Parasitol 1989;33:135-42.

[19] Gibson WC, Borst P. Size-fractionation of the small chromosomes of Trypanozoon and Nannomonas trypanosomes by pulsed field gradient gel electrophoresis. Mol Biochem Parasitol 1986;18:127-40.

[20] Van der Ploeg LHT, Cornelissen AWCA, Barry JD, Borst P. Chromosomes of Kinetoplastida. EMBO J 1984;3:3109-15.

[21] Jenni L, Marti S, Schweizer J, et al. Hybrid formation between African trypanosomes during cyclical transmission. Nature 1986;322:173-5.

[22] Bernards A, Michels PAM, Lincke CR, Borst P. Growth of chromosome ends in multiplying trypanosomes. Nature 1983;303:592-7.

[23] Van der Ploeg LHT, Liu AYC, Borst P. Structure of the growing telomeres of trypanosomes. Cell 1984;36:459-68.

[24] Horn D. Telomere maintenance and length regulation in Trypanosoma brucei. EMBO J 2000;19:2332-9.

[25] Patnaik PK, Axelrod N, Van der Ploeg LHT, Cross GAM. Artificial linear mini-chromosomes for Trypanosoma brucei. Nucl Acids Res 1996;24:668-75.

[26] Pays E, Laurent M, Delinte K, van Meirvenne N, Steinert M. Differential size variations between transcriptionally active and inactive telomeres of Trypanosoma brucei. Nucl Acids Res 1983;11:8137-47.

[27] Lee MG, EY, Axelrod N. Construction of trypanosome artificial mini-chromosomes, Nucl. Acids Res. 1995; 23: 4893-4899. 